\title{
Topic Modeling of Social Networking Service Data on Occupational Accidents in Korea: Latent Dirichlet Allocation Analysis
}

\author{
Kyoung-Bok Min ${ }^{1}$, PhD; Sung-Hee Song ${ }^{1}$, BA; Jin-Young Min ${ }^{2}, \mathrm{PhD}$ \\ ${ }^{1}$ College of Medicine, Seoul National University, Seoul, Republic of Korea \\ ${ }^{2}$ Institute of Health and Environment, Seoul National University School of Public Health, Seoul, Republic of Korea
}

\section{Corresponding Author:}

Jin-Young Min, $\mathrm{PhD}$

Institute of Health and Environment

Seoul National University School of Public Health

Gwanak-ro 1, Gwanak-gu

Seoul, 08826

Republic of Korea

Phone: 828802759

Email: yaemin00@snu.ac.kr

\section{Abstract}

Background: In most industrialized societies, regulations, inspections, insurance, and legal options are established to support workers who suffer injury, disease, or death in relation to their work; in practice, these resources are imperfect or even unavailable due to workplace or employer obstruction. Thus, limitations exist to identify unmet needs in occupational safety and health information.

Objective: The aim of this study was to explore hidden issues related to occupational accidents by examining social network services (SNS) data using topic modeling.

Methods: Based on the results of a Google search for the phrases occupational accident, industrial accident and occupational diseases, a total of 145 websites were selected. From among these websites, we collected 15,244 documents on queries related to occupational accidents between 2002 and 2018. To transform unstructured text into structure data, natural language processing of the Korean language was conducted. We performed the latent Dirichlet allocation (LDA) as a topic model using a Python library. A time-series linear regression analysis was also conducted to identify yearly trends for the given documents.

Results: The results of the LDA model showed 14 topics with 3 themes: workers' compensation benefits (Theme 1), illicit agreements with the employer (Theme 2), and fatal and non-fatal injuries and vulnerable workers (Theme 3). Theme 1 represented the largest cluster (52.2\%) of the collected documents and included keywords related to workers' compensation (ie, company, occupational injury, insurance, accident, approval, and compensation) and keywords describing specific compensation benefits such as medical expense benefits, temporary incapacity benefits, and disability benefits. In the yearly trend, Theme 1 gradually decreased; however, other themes showed an overall increasing pattern. Certain queries (ie, musculoskeletal system, critical care, and foreign workers) showed no significant variation in the number of queries.

Conclusions: We conducted LDA analysis of SNS data of occupational accident-related queries and discovered that the primary concerns of workers posting about occupational injuries and diseases were workers' compensation benefits, fatal and non-fatal injuries, vulnerable workers, and illicit agreements with employers. While traditional systems focus mainly on quantitative monitoring of occupational accidents, qualitative aspects formulated by topic modeling from unstructured SNS queries may be valuable to address inequalities and improve occupational health and safety.

(J Med Internet Res 2020;22(8):e19222) doi: 10.2196/19222

\section{KEYWORDS}

topic modeling; occupational accident; social media; knowledge; workplace; accident; model; analysis; safety 


\section{Introduction}

Occupational health and safety are fundamental components of a good work environment [1]. They are relevant not only to increased productivity but also to the moral and legal responsibilities of both employees and employers [1]. Although many strategies and programs promote occupational safety and health, fatal and nonfatal accidents in the work environment remain a global problem [1,2]. Occupational accidents include any injury, disease, or death arising through the course of employment [2]. The International Labor Organization estimates that 2.78 million workers die each year from occupational injuries, and 374 million workers suffer nonfatal work-related injuries and illnesses [2].

Occupational accident statistics vary from country to country due to differences in coverage, definitions, and classifications; data divided into fatal and nonfatal rates of occupational accidents provide similar perspectives on the risks related to occupational safety and health [2]. The paradox of low levels of nonfatal injuries but high rates of fatal accidents is observed in many countries, including South Korea [3,4]. In 2017, the rate of fatal occupational injuries reached 1.12 per 10,000 Korean workers; however, the rate of nonfatal occupational injuries was 0.54 per 100 workers [5]. This paradox may be rooted in underreporting or covering up of nonfatal occupational accidents to avoid reprisal or stigma at work or due to a perception that an injury is minor or simply part of the job $[3,6]$. Thus, conventional record keeping of occupational accidents may simply fail to provide the actual occupational injury rates. Furthermore, there are limitations to identifying unmet needs in occupational safety and health information, resources, and interventions for injured workers.

Due to the rapid spread of smart devices and mobile internet services, social network services (SNSs) are changing the ways in which people interact with other people who have similar interests, thoughts, services, or desires [7-9]. SNS users can also engage in knowledge sharing about specific topics (ie, academic, medical, and legal issues) [7-9]. People with work-related injuries or illnesses differ from other SNS users in how they seek professional advice and information over the internet. Employers sometimes present obstacles to injured workers or minimize their benefits, and the process of approving workers' compensation benefits is time-consuming and frustrating. Therefore, it is inevitable that injured workers will seek legal and technical information and discuss their challenges through SNSs.

We believe that SNS data do not show the entire picture of occupational accidents but are useful for discovering hidden aspects of these accidents that are not captured by conventional surveillance. In this study, by applying topic modeling techniques to SNS data, we explored issues regarding occupational accidents that are not shared or discussed publicly but are nonetheless important.

\section{Methods}

The data upon which our study is based were obtained using Google's Korean-language search engine (Figure 1). Using the results of a Google search for the phrases occupational accident, industrial accident, and occupational diseases, a total of 129,000 webpages were initially selected. Next, 87,800 webpages were excluded from the initial sites by applying the keyword workers compensation. We further filtered the keywords certified labor attorneys, doctors, and other attorneys, and we excluded webpages corresponding to news, laws, and regulations. Ultimately, 355 websites were identified after applying the filter. We subsequently narrowed down our selection to 210 expert counseling and knowledge-sharing websites on which injured workers and their families could discuss difficulties related to treatment and compensation after occupational accidents and could seek answers and advice from experts. Finally, 145 websites were deemed eligible for use in the current study, excluding those that were suspended or contained fewer than 10 posts. We used web crawlers to gather website data from between 2002 and 2018; a total of 23,076 documents were collected from 145 websites. These documents were composed of full-text documents (articles or posts), comments, and blogs. From among these websites, 7832 duplicate documents were excluded. Finally, 15,244 documents were subjected to further analysis. 
Figure 1. Flowchart of the identification of 145 websites (as of May 2019).

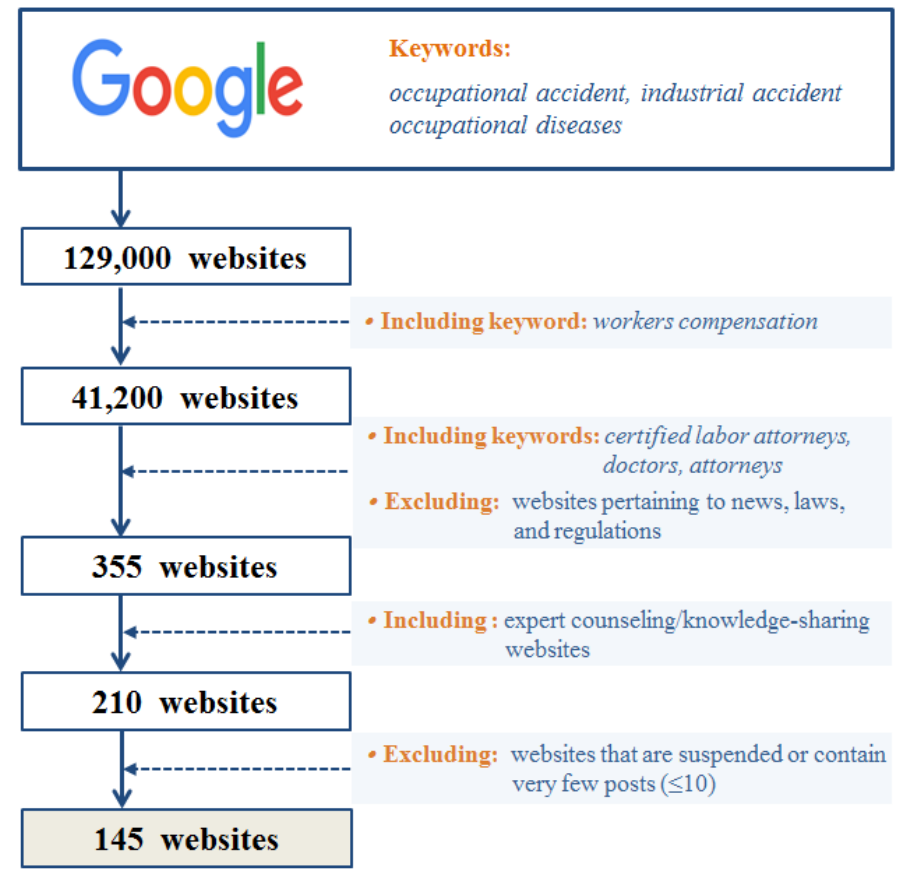

Prior to topic modeling analysis, the various documents were transformed into a structured form by text preprocessing. Natural language processing (NLP) was performed using KoNLPy, which is an open-source morphological analyzer for Korean processes based on the Python package [10]. Lexical analysis was performed by sentence splitting followed by tokenizing. We removed unnecessary components such as unnecessary white spaces, punctuation, special characters, and stop words such as "a," "the," and "it" from the unstructured data. To convert the sentences from the documents into words, tokenization was conducted using $\mathrm{MeCab}$ [11], an open-source morphological analyzer and part-of-speech tagger modified for the Korean language.

With keywords created through NLP, a term-document matrix was created to evaluate the importance of a word in a document. We used the term frequency-inverse document frequency (TF-IDF) technique [12], in which the term frequency measures how often a word appears in a given document divided by the total number of words while the inverse document frequency measures how frequently a word appears in all documents divided by the number of documents that contain the word. Thus, a word with a high TF-IDF score is distinctly frequent in a given document compared with other documents in the set. In this way, a term-document matrix of 35,315 words was generated from 15,244 documents. We created a co-occurrence network for high-frequency words in the given documents.

Topic modeling is a machine learning technique that is used to determine the abstract topics discussed in a given text [13]. Topic modeling is recognized as a standard methodology with high performance and convenience; it has been suggested as an alternative method of solving problems of rareness, synonyms, multiplicity, and semantic hierarchy that occur in existing word frequency analyses [13]. Latent Dirichlet allocation (LDA), a generative probabilistic model of a corpus, is a commonly used topic model. LDA assumes sparse Dirichlet prior distributions, encoding the intuition that the probability distribution of words in a topic is skewed so that only a small set of words have high probability $[14,15]$. In a collection of documents (D) including a word (W) and a preselected number of topics (K), LDA calculates two probabilities: the probability of words in document $\mathrm{d}$ assigned to topic $\mathrm{t}, \mathrm{P}(\mathrm{t} \mid \mathrm{d})$, and the probability of topic $t$ in all the documents in a set for word $\mathrm{w}, \mathrm{P}(\mathrm{w} \mid \mathrm{t})$. The prior distributions of $\mathrm{P}(\mathrm{t} \mid \mathrm{d})$ and $\mathrm{P}(\mathrm{w} \mid \mathrm{t})$ are defined by the hyperparameters $\alpha$ and $\beta$. Gibbs sampling is used to assess distribution over topics and distribution over words for each document $[13,16]$. We used LDA in the Gensim library in Python for topic modeling. We set $\lambda$ as 0.6 and ran the LDA with 3000 Gibbs sampling iterations. The optimal number of topics was based on perplexity and coherence (Supplemental Figure 1 in Multimedia Appendix 1). Perplexity is a method of evaluating how well a probability distribution can predict a held-out sample [17]. The smaller the change in the perplexity value, the better the probabilistic model. Topic coherence measures the score of a single topic by calculating the degree of similarity between the top $\mathrm{N}$ words of a topic [18]. The higher the score, the easier it is to choose the appropriate number of topics. Because there are variations in the interpretation of the quality of topics among perplexity, coherence, and human judgment [15], we further evaluated the manually formulated topics by varying the number of topics of the LDA model. Finally, we determined that 14 was a reasonable number of topics to discover hidden structures in the text body. Each topic was represented by a set of several keywords and was named by the authors of this report, who included a physician specializing in occupational medicine. These topics were categorized into three themes based on the LDA plot and the authors' interpretation. For each topic, we performed time-series linear regression analysis with SAS statistical software (SAS Institute) by using the AUTOREG procedure to identify trends by year. The years were taken as independent variables, and the dependent variables were the average weight values for each 
topic by year. We classified the topics as "hot" if the regression coefficient was positive or as "cold" if the coefficient was negative, taking a 5\% significance level.

\section{Results}

Table 1 shows the numbers of documents related to occupational accidents collected on the internet by year. From the 145 websites, a total of 15,244 documents were identified during the study period (2002 to 2018). The number of documents fluctuated over time. In the first three years, from 2002 to 2005 , about 100 documents $(0.6 \%$ of the total documents) were identified. Occupational accident queries were very frequent in 2011 but then declined briefly. After 2012, the number of queries surged again, resulting in an average of over 1500 queries per year.

Figure 2 shows the co-occurrence network of the high frequency keywords in the set of documents. Using keyword analysis, we investigated the degrees of connection between major keywords.
The green lines indicate the connections between keywords; the darker the shade of green, the more connected the words. Among the top 100 keywords derived from TF-IDF, the keywords related to workers' compensation showed the highest frequencies. These keywords were company, process, salary, insurance, request, treatment, and occupational accidents. Keywords related to occupational injuries, such as hospitals, surgical operation, rates, finger, and hospitalization, were also highly frequent. These high-frequency words linked to workers' compensation and occupational injuries were interconnected with many other words.

Figure 3 presents the results of the LDA model; 14 topics were formulated using LDA. This set was plotted in a 2D plan along the transverse (PC1) and longitudinal (PC2) axes. Each topic was displayed as a circle, and the overall prevalence was calculated as the areas of the circles. The centers of each topic were determined by computing the distance between topics. The 14 topics were manually classified into 3 themes.

Table 1. Numbers of documents related to occupational accidents by year $(\mathrm{N}=15,244)$.

\begin{tabular}{ll}
\hline Year & Documents, $\mathrm{n}(\%)$ \\
\hline 2002 & $9(0.06)$ \\
2003 & $19(0.12)$ \\
2004 & $34(0.22)$ \\
2005 & $32(0.21)$ \\
2006 & $104(0.68)$ \\
2007 & $307(2.01)$ \\
2008 & $192(1.26)$ \\
2009 & $1148(7.53)$ \\
2010 & $699(4.59)$ \\
2011 & $480(3.15)$ \\
2012 & $1602(10.51)$ \\
2013 & $1977(12.97)$ \\
2014 & $1774(11.64)$ \\
2015 & $1754(11.51)$ \\
2016 & $1508(9.89)$ \\
2017 & $1934(12.69)$ \\
2018 & $1671(10.96)$ \\
\hline
\end{tabular}


Figure 2. Co-occurrence network of 100 high-frequency keywords in documents related to occupational accidents.

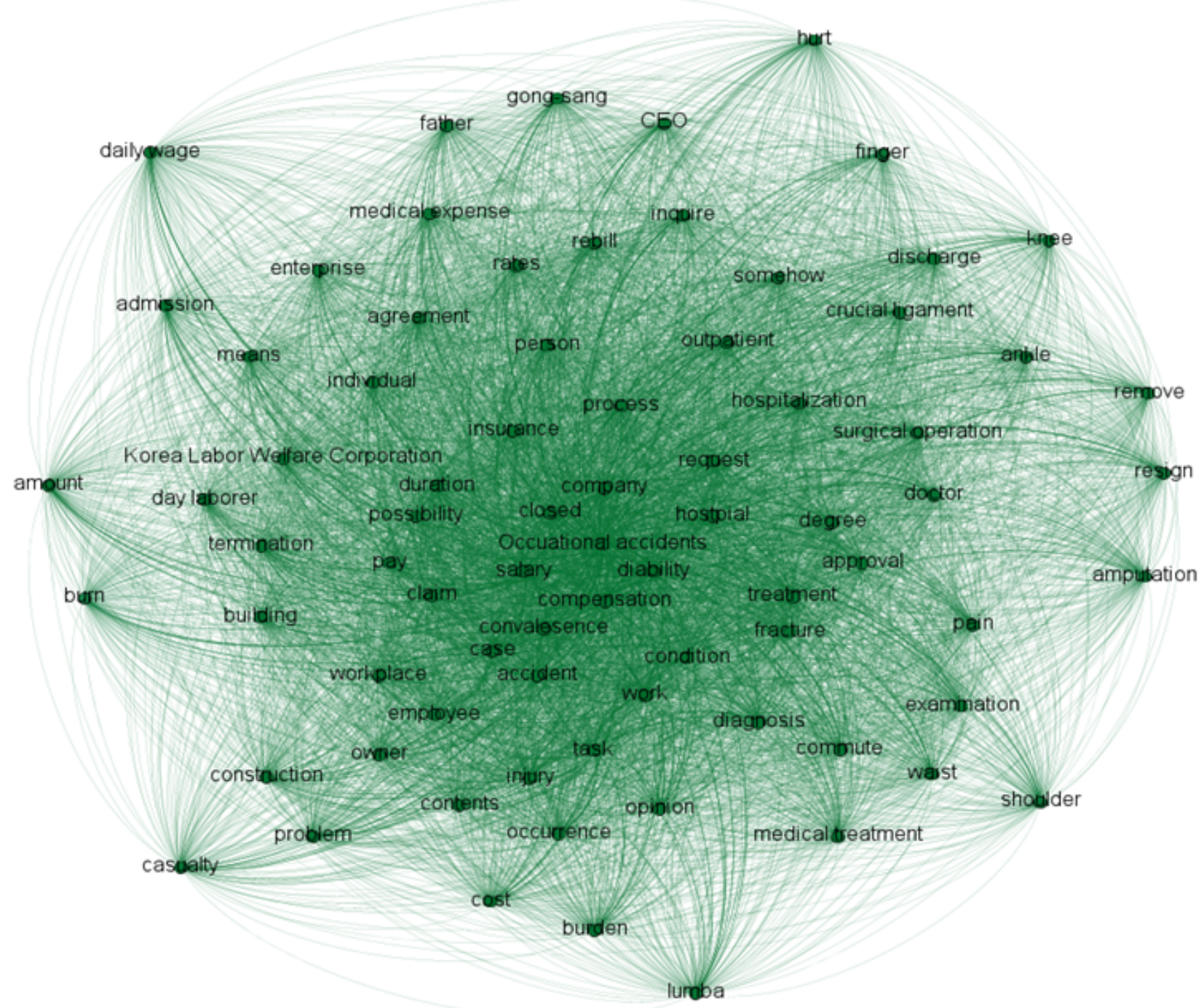

Figure 3. Overview of the formulation of 14 topics and 3 themes with latent Dirichlet allocation topic modeling.

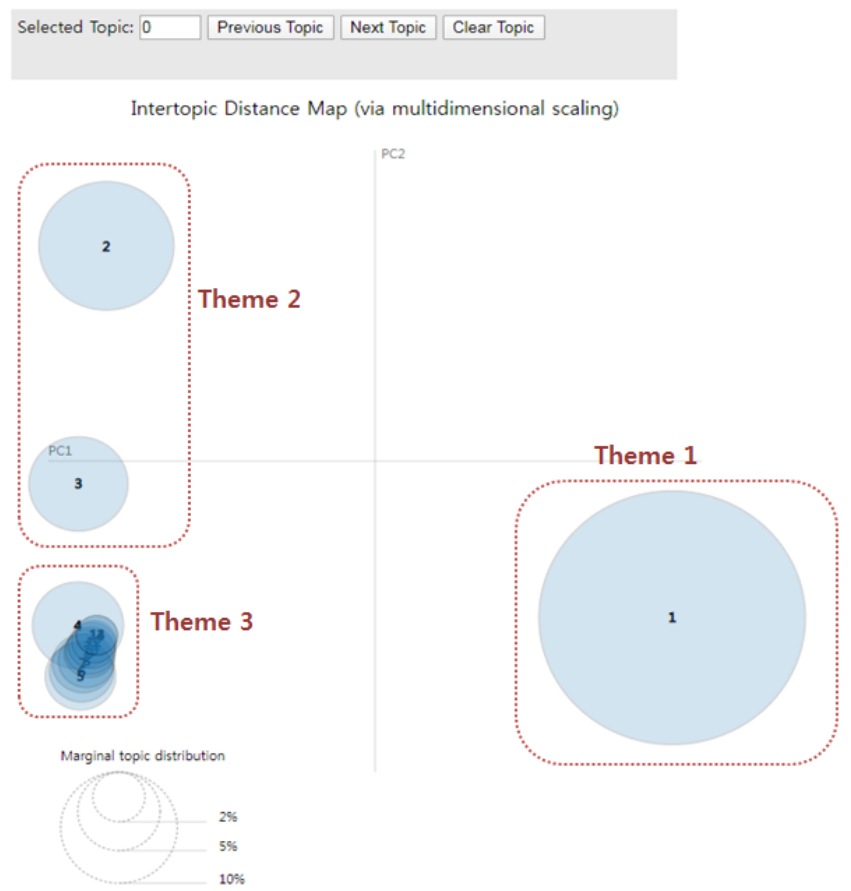


Table 2 shows the formulated topics and keywords from the LDA models, including the themes, topics, subjects, keywords, and topic proportions (ie, percentages). A total of 14 topics were divided into 3 themes. The topic proportion was defined as the proportion of words in a document that belong to a topic; this measurement indicates the main topics in a document. Herein, Topic 1 appeared the most frequently, at $52.2 \%$, and was designated Theme 1 (workers' compensation benefits). Many of the keywords for Topic 1 were associated with workers' compensation (ie, company, process, occupational injury, insurance, and compensation), medical expense benefits (ie, hospital, surgical operation, medical treatment, hospitalization, and convalescence), temporary incapacity benefits (ie, salary, request, approval, workplace, and shutdown), and disability benefits (ie, possibility, rates, disability, fracture, and impairment). Topics 2 and 3, which totaled approximately $20.6 \%$ of all topics, were categorized as Theme 2, illicit agreement (called gong-sang in Korean) with the employer. These two topics contained keywords related to gong-sang: specifically, related to the musculoskeletal system of Topic 2 (ie, gong-sang, disk, rupture, MRI, lumbar, traffic accident, backbone, orthopedics, surgical procedure, and X-ray) and physical trauma of Topic 3 (ie, gong-sang, thumb, metal pin, reattachment, suture, tendon, bruise, scar, stiches, and infection). The remaining Topics $4-14$, approximately $27.2 \%$ of the total, included keywords describing fatal and nonfatal injuries and vulnerable workers, Theme 3. Specifically, Topics 4, 5, 6, 9, and 10 implied keywords related to fatal and nonfatal injuries, such as critical care (Topic 4), fatal accident (Topic 5), lower extremity injury (Topic 6), fracture (Topic 9), and labor-management conflict (Topic 10). The remaining topics included keywords describing vulnerable workers, such as restaurant workers (Topic 7), construction workers (Topic 8), vulnerable jobs (Topic 11), student workers (Topic 12), and foreign workers (Topic 13). In addition, Topic 14 included words such as hearing, hearing loss, hepatocirrhosis, elderly, soft tissue, sudden, and garbage man.

Table 3 displays the regression coefficients and Figure 4 displays the heat map for the yearly changes in interest in the 14 topics from the LDA models. The largest topic was Topic 1, relating to workers' compensation benefits; interest in this topic significantly decreased over time. In contrast, interest in Topic 2 (musculoskeletal system), Topic 4 (critical care), and Topic 13 (foreign workers) continued over time without significant changes. Most of the topics (Topic 3, Topics 5 to 12, and Topic 14) showed significant increases over time $(P<.05)$. 
Table 2. Themes, topics, and keywords formulated by latent Dirichlet allocation topic modeling.

\begin{tabular}{ll}
\hline Theme and topics & Keywords \\
\hline Theme 1: Workers' compensation benefits & \\
Topic 1a: Workers' compensation & company, process, occupational injury, insurance, compensation \\
Topic 1b: Medical expense benefits & hospital, surgical operation, medical treatment, hospitalization, convalescence \\
Topic 1c: Temporary incapacity benefits & salary, request, approval, workplace, shutdown \\
Topic 1d: Disability benefits & possibility, rates, disability, fracture, impairment
\end{tabular}

\section{Theme 2: Illicit agreement (gong-sang) with employer}

Topic 2: Musculoskeletal system

Topic 3: Physical trauma gong-sang, disk, rupture, $M R I^{\mathrm{a}}$, lumbar, traffic accident, backbone, orthopedics, surgical procedure, $X$-ray

gong-sang, thumb, metal pin, reattachment, suture, tendon, bruise, scar, stiches, infection

\section{Theme 3: Fatal and nonfatal injuries and vulnerable workers}

Topic 4: Critical care

Topic 5: Fatal accident

Topic 6: Lower extremity injury

Topic 7: Restaurant workers

Topic 8: Construction workers

Topic 9: Fracture

Topic 10: Labor-management conflict

Topic 11: Vulnerable jobs

Topic 12: Student workers

Topic 13: Foreign workers

Topic 14: Others bleeding, intensive care unit, nothing, whole life, hospital room, tear, infection die, bereaved, police, statement, rolled, autopsy, law

cast, myoelectric, numbness, stabbed, foot, planta pedis, bumped

service, kitchen, owner, sick, piece of meat, hotel, McDonald

fall, construction site, carelessness, Achilles tendon, builders, license, cement 2.3

open fracture, tumble, rib, incisura, cervical vertebrae, muscular pain, ventral 1.9 root

penalty, headquarters, Labor office, disgusted, civil and criminal, credit, tape- 1.8 recording

construction workers, facilities, line, factory, guard, service industry, fast food 1.7

disadvantage, part-timers, manager, professor, college student, class, atten- 1.4 dance

identity, visa, sojourn, local, Vietnam, Korea, reentry

hearing, hearing loss, hepatocirrhosis, elderly, soft tissue, sudden, garbage man

${ }^{\mathrm{a}}$ MRI: magnetic resonance imaging. 
Table 3. Regression coefficients of the yearly changes in interest in the 14 topics.

\begin{tabular}{llll}
\hline Theme and topic & Estimate & $P$ value & Cold/Hot \\
\hline Theme 1 & & & Cold \\
Topic 1: Workers' compensation benefits & -0.2885 & $<.001$ & - $^{\mathrm{a}}$ \\
Theme 2 & -0.0665 & .10 & Hot \\
Topic 2: Musculoskeletal system & 0.1194 & .01 & - \\
Topic 3: Physical trauma & & Hot \\
Theme 3 & -0.0042 & .90 & Hot \\
Topic 4: Critical care & 0.0569 & .01 & Hot \\
Topic 5: Fatal accident & 0.0207 & .08 & Hot \\
Topic 6: Lower extremity injury & 0.0465 & $<.001$ & Hot \\
Topic 7: Restaurant workers & 0.0324 & $<.001$ & Hot \\
Topic 8: Construction workers & 0.0178 & .004 & Hot \\
Topic 9: Fracture & 0.0261 & $<.001$ & Hot \\
Topic 10: Labor-management conflict & 0.0139 & .002 & Hot \\
Topic 11: Vulnerable jobs & 0.0208 & .001 & \\
Topic 12: Student workers & -0.0040 & 0.0086 & \\
Topic 13: Foreign workers & &
\end{tabular}

a_: no significant variation.

Figure 4. Heat map of the yearly changes in interest in the 14 topics.

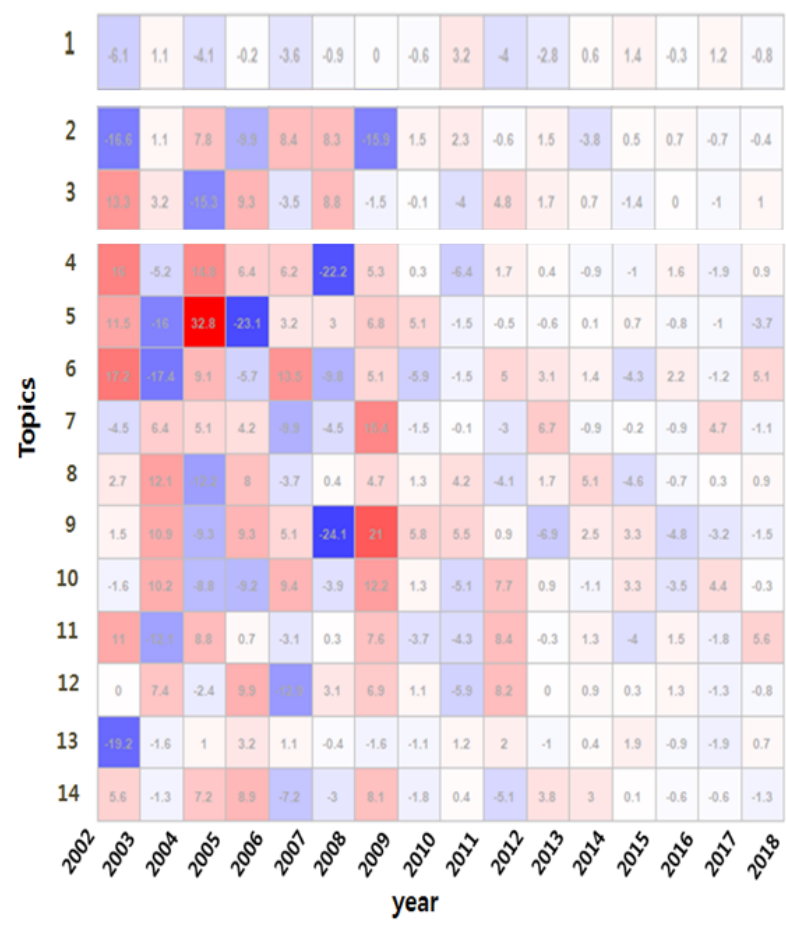

\section{Discussion}

\section{Principal Findings}

Occupational accidents are a major public health challenge [1]. Although surveillance systems have been constructed to monitor the mortality and morbidity of occupational accidents, the surveillance data may be inaccurate due to underreporting or covering up of injuries and illnesses [6,19]. This impedes proper evaluation of the magnitude of health and safety problems in the workplace, which reduces protection of workers from workplace hazards, identification of risks, and implementation of needed interventions. Our study investigated hidden issues in occupational accidents observed on the internet. We collected 
data from SNSs on occupational accident-related queries and analyzed them using the LDA topic model. The LDA analysis extracted a total of 14 topics, which we clustered into 3 themes: workers' compensation benefits (Theme 1), illicit agreements with the employer (Theme 2), and fatal and nonfatal injuries and vulnerable workers (Theme 3 ).

The largest share of $52.2 \%$ of the collected documents concerned workers' compensation benefits; this was classified as Theme 1. In general, if a worker is injured while working, they do not or cannot work for a certain period of time. This not only leads to the burden of medical expenses but also considerably impacts the livelihood of the worker and their family due to the suspension of household income. To ensure social security as a collective measure against injuries, disease, and death, the Korean government implemented the Industrial Accident Compensation Insurance Act 2015, in which all workers are required to participate. The Industrial Accident Compensation Insurance Act pays compensation (hereafter referred to as workers' compensation benefits) such as medical care benefits, temporary layoff benefits, and disability benefits to workers who are injured or disabled at work [20,21]. Although Korean workers who require more than three days of treatment are covered by the Industrial Accident Compensation Insurance Act, it has been found that the approval process for workers' compensation benefits can be protracted and difficult because insurers and employers sometimes attempt to limit injured workers' benefits [20]. Therefore, the primary concern of workers suffering from occupational injuries or diseases may focus on obtaining compensation benefits through legal procedures rather than how the occupational injury or disease affects them. That is, affected workers need advice from legal experts (such as certified labor attorneys and other attorneys) regarding the type of compensation they are eligible for. Due to these affected workers' needs, Theme 1 is the most frequently mentioned subject throughout the entire industrial accident query. As shown in Table 2, Theme 1 is composed of Topic 1, which includes keywords related to workers' compensation such as company, process, request, benefits, occupational injury, insurance, accident, approval, and compensation. In addition, it includes keywords describing the specific benefits of industrial accident compensation, namely medical expense benefits (ie, hospital, surgical operation, medical treatment, hospitalization, and convalescence); temporary incapacity benefits (ie, salary, request, approval, workplace, and shutdown); and disability benefits (ie, possibility, rates, disability, fracture, and impairment).

The second-largest cluster was Theme 3, fatal and nonfatal injuries and vulnerable workers, with 28.2 percent of documents. When we considered occupational accidents and industrial accident compensation, we found that the major concern of injured or disabled workers during work was whether they could be beneficiaries of industrial accident compensation. Although numerous injuries and illnesses are known to result from occupational causes [1,22], establishing a causal relationship between work factors and injuries is a prerequisite for the approval of industrial accident compensation. Therefore, the affected workers appear to have described their occupationally related injuries on SNSs, and on the same platforms, they asked whether their accidents or illnesses would constitute legitimate industrial accidents. In Theme 3, topics 4, 5, 6, 9, 10, and 14 encompassed keywords describing fatal and nonfatal occupation-related injuries (ie, bleeding, die, cast, fracture, incisura, and hearing loss). Another issue in Theme 3 is the employment status of affected workers. Workers' employment status affects health and causes safety disparities, which is exacerbated by unregulated and unsafe workplaces [23-25]. International reviews have found that occupational injury rates for nonstandard and temporary workers are greater than those for permanent workers [26,27]. Nonregular workers such as part-time workers, temporary contract workers, and dispatched workers may be excluded from social insurance and corporate welfare programs, and they may receive no compensation when they are affected by industrial accidents. In fact, many workers on fixed terms are not covered by workers' compensation [28]. Keywords related to vulnerable workers (ie, service, hotel, construction workers, guard, fast food, and Vietnam) were found in topics $3,7,8,11,12$, and 13 in Theme 3.

Finally, Theme 2 accounted for the smallest percentage (20.6\%) of the collected documents. The subject "illicit agreement" (gong-sang in Korean) in Theme 2 refers to cases where injured employees negotiate settlements separately (ie, beyond the legal purview) with their employers instead of legally declaring their accidents and pursuing workers' compensation benefits [29]. Although injuries to the musculoskeletal system (Topic 2) and physical trauma (Topic 3) were the most common types of occupational injury, they are considered to be mild injuries, and it can be difficult to prove that they are work-related. Therefore, workers' compensation claims for these injuries are rarely approved. Thus, when workers suffer from musculoskeletal disease or physical trauma (ie, disk, rupture, bruise, and reattachment), they often enter into illicit agreements with their employers to receive payment from the companies.

We also performed a time-series analysis to investigate the annual changes in themes and topics between 2002 and 2018. Typical queries regarding the compensation for occupational accidents (seen in Theme 1) were found to decrease over time, whereas most queries related to themes 2 and 3 increased. Notably, certain queries regarding the musculoskeletal system (Topic 2 in Theme 2), critical care (Topic 4 in Theme 3), and foreign workers (Topic 13 in Theme 3) showed no significant variation in the number of queries during the study period (2002 to 2018). This appears to indicate that those topics surface consistently every year in discussions surrounding occupational accidents.

LDA topic modeling is a method that is used to identify the underlying topics contained in unstructured text data. This method is used widely in topic detection in medicine, marketing research, political science, and linguistics [16,30,31]. Our study is the first to use LDA techniques to discover latent issues related to occupational accidents. Based on web-based and unstructured web-based documents, we obtained novel insights into the unfulfilled needs of industrial accident workers and the information they sought via expert consultation as well as the occupations that are vulnerable to industrial accidents. 


\section{Limitations}

We should mention several important limitations regarding the methods used in this study. The main critique here relates to the instability of topic distribution and interpretation. Because topic modeling is sensitive to input data and analysis, changes (such as adding new documents and implementing text mining algorithms such as tokenization and stemming) can generate completely different topics. Therefore, the topics are often an amalgam, and it is difficult to assign truth to the interpretation and validation in the given corpus [32]. In addition, we focused on SNS data from experts (ie, certified labor attorneys, other attorneys, and physicians) on counseling websites between 2002 and 2018 with the aim of exploring latent issues in occupational accidents. However, depending on the SNS data source and time period collected, different topics and themes can be derived from our results. Therefore, our results may not genuinely represent the overall view of occupational accidents in our country. Moreover, applying our results to other countries with distinct industrial accident laws and regulations would require considerable attention.

\section{Conclusion}

We conducted LDA analysis with SNS data related to occupational accidents and discovered that the primary concerns of workers posting about occupational injuries and diseases were workers' compensation benefits, fatal and nonfatal injuries and vulnerable workers, and illicit agreements with employers. While traditional systems focus mainly on quantitative monitoring of occupational accidents, qualitative aspects formulated by topic modeling from unstructured SNS queries may be valuable for providing practical knowledge and information to the affected workers. This approach may be useful to address inequality among workers and improve occupational health and safety.

\section{Acknowledgments}

This work was supported by a National Research Foundation of Korea (NRF) grant funded by the Korean government (No. NRF-2017R1E1A1A01078235).

\section{Conflicts of Interest}

None declared.

\section{Multimedia Appendix 1}

Supplementary table and figure.

[DOCX File, 72 KB-Multimedia Appendix 1]

\section{References}

1. Alli BO. Fundamental Principles of Occupational Health and Safety, Second Edition. Geneva: International Labour Office; 2008.

2. Global Estimates of Occupational Accidents and Work-Related Illnesses 2017. Singapore: Workplace Safety and Health Institute; Sep 2017.

3. Mendeloff J, Burns R. States with low non-fatal injury rates have high fatality rates and vice-versa. Am J Ind Med 2013 May;56(5):509-519. [doi: 10.1002/ajim.22047] [Medline: 22473632]

4. Rhee KY, Choe SW, Kim YS, Koo KH. The trend of occupational injuries in Korea from 2001 to 2010. Saf Health Work 2013 Mar;4(1):63-70 [FREE Full text] [doi: 10.5491/SHAW.2013.4.1.63] [Medline: 23515324]

5. Statistics on industrial accident in 2017. Korean Statistical Information Service. South Korea; 2017. URL: http://www. index.go.kr [accessed 2020-06-10]

6. Moore JT, Cigularov KP, Sampson JM, Rosecrance JC, Chen PY. Construction workers' reasons for not reporting work-related injuries: an exploratory study. Int J Occup Saf Ergon 2013;19(1):97-105. [doi: 10.1080/10803548.2013.11076969] [Medline: 23498712]

7. Bender JL, Cyr AB, Arbuckle L, Ferris LE. Ethics and Privacy Implications of Using the Internet and Social Media to Recruit Participants for Health Research: A Privacy-by-Design Framework for Online Recruitment. J Med Internet Res 2017 Apr 06;19(4):e104 [FREE Full text] [doi: 10.2196/jmir.7029] [Medline: 28385682]

8. Chan WS, Leung AY. Use of Social Network Sites for Communication Among Health Professionals: Systematic Review. J Med Internet Res 2018 Mar 28;20(3):e117. [doi: 10.2196/jmir.8382] [Medline: 29592845]

9. Dagan N, Beskin D, Brezis M, Reis BY. Effects of Social Network Exposure on Nutritional Learning: Development of an Online Educational Platform. JMIR Serious Games 2015;3(2):e7 [FREE Full text] [doi: 10.2196/games.4002] [Medline: 26441466]

10. Park E, Cho S. KoNLPy: Korean natural language processing in Python. 2014 Oct 10 Presented at: Paper presented at the 26th Annual Conference on Human \& Cognitive Language Technology; Oct 2014; Chuncheon, Korea.

11. MeCab: Yet Another Part-of-Speech and Morphological Analyzer. Webpage in Japanese. GitHub. URL: http://taku910. github.io/mecab/ [accessed 2020-07-26]

12. Jeffrey D, Ullman J, Jur L. Mining of Massive Datasets. Stanford University Infolab. URL: http://infolab.stanford.edu/ ullman/mmds/ch9.pdf [accessed 2020-06-10] 
13. Blei D, Ng A, Jordan M. Latent Dirichllocation. J Mach Learn Res 2003;3:993-1022.

14. Blei DM. Probabilistic topic models. Commun ACM 2012 Apr;55(4):77-84. [doi: 10.1145/2133806.2133826]

15. Chang J, Boyd-Graber J, Wang C, Gerrish S, Blei DM. Reading tea leaves: How humans interpret topic models. Neural Information Processing Systems. 2009. URL: https://papers.nips.cc/paper/

3700-reading-tea-leaves-how-humans-interpret-topic-models.pdf [accessed 2020-06-10]

16. Huang M, ElTayeby O, Zolnoori M, Yao L. Public Opinions Toward Diseases: Infodemiological Study on News Media Data. J Med Internet Res 2018 May 08;20(5):e10047 [FREE Full text] [doi: 10.2196/10047] [Medline: 29739741]

17. Jacobi C, van Atteveldt W, Welbers K. Quantitative analysis of large amounts of journalistic texts using topic modelling. Digital Journalism 2015 Oct 13;4(1):89-106. [doi: 10.1080/21670811.2015.1093271]

18. Newman D, Jey HL, Karl G, Timothy B. Automatic Evaluation of Topic Coherence. 2010 Jun 2 Presented at: Human Language Technologies: The 2010 Annual Conference of the North American Chapter of the Association for Computational Linguistics; June 2-4, 2010; Los Angeles, CA URL: https://www.aclweb.org/anthology/N10-1012.pdf

19. Lim S, Yoon J, Rhie J, Bae SW, Kim J, Won J. The Relationship between Free Press and Under-Reporting of Non-Fatal Occupational Injuries with Data from Representative National Indicators, 2015: Focusing on the Lethality Rate of Occupational Injuries among 39 Countries. Int J Environ Res Public Health 2018 Dec 14;15(12):2856 [REE Full text] [doi: 10.3390/ijerph15122856] [Medline: 30558139]

20. Kang S, Kwon O. Occupational Injury Statistics in Korea. Saf Health Work 2011 Mar;2(1):52-56. [doi: 10.5491/shaw.2011.2.1.52]

21. Kim JG, Duk YJ. Development and Operation of Workers' Compensation Insurance Scheme in S. Korea. Ministry of Employment and Labor, Republic of Korea. 2012. URL: https://www.moel.go.kr/english/download eng. jsp?type $=\&$ file $=12664$ 2.pdf $[$ accessed 2020-06-10]

22. LaDou J. International occupational health. Int J Hyg Environ Health 2003 Aug;206(4-5):303-313. [doi: 10.1078/1438-4639-00226] [Medline: 12971685]

23. Min J, Park S, Hwang SH, Min K. Disparities in precarious workers' health care access in South Korea. Am J Ind Med 2016 Dec;59(12):1136-1144. [doi: 10.1002/ajim.22658] [Medline: 27699816]

24. Min KB, Park SG, Song JS, Yi KH, Jang TW, Min JY. Subcontractors and increased risk for work-related diseases and absenteeism. Am J Ind Med 2013 Nov;56(11):1296-1306. [doi: 10.1002/ajim.22219] [Medline: 23794385]

25. Siqueira CE, Gaydos M, Monforton C, Slatin C, Borkowski L, Dooley P, et al. Effects of social, economic, and labor policies on occupational health disparities. Am J Ind Med 2014 May;57(5):557-572 [FREE Full text] [doi: 10.1002/ajim.22186] [Medline: 23606055]

26. Nicholson VJ, Bunn TL, Costich JF. Disparities in work-related injuries associated with worker compensation coverage status. Am J Ind Med 2008 Jun;51(6):393-398. [doi: 10.1002/ajim.20565] [Medline: 18381597]

27. Quinlan M. The effects of non-standard forms of employment on worker health and safety. International Labour Organization. Geneva: International Labour Office; 2015. URL: https://www.ilo.org/wcmsp5/groups/public/---ed_protect/---protrav/ ---travail/documents/publication/wcms 443266.pdf [accessed 2020-08-09]

28. Employment arrangements: Improved outreach could help ensure proper worker classification. United States Government Accountability Office. 2006. URL: https://www.gao.gov/new.items/d06656.pdf [accessed 2020-08-09]

29. Min J, Song S, Kim H, Min K. Mining Hidden Knowledge About Illegal Compensation for Occupational Injury: Topic Model Approach. JMIR Med Inform 2019 Sep 26;7(3):e14763 [FREE Full text] [doi: 10.2196/14763] [Medline: $\underline{31573948]}$

30. Hao H, Zhang K. The Voice of Chinese Health Consumers: A Text Mining Approach to Web-Based Physician Reviews. J Med Internet Res 2016 May 10;18(5):e108 [FREE Full text] [doi: 10.2196/jmir.4430] [Medline: 27165558]

31. Jelodar H, Wang Y, Yuan C, Feng X, Jiang X, Li Y, et al. Latent Dirichlet allocation (LDA) and topic modeling: models, applications, a survey. Multimed Tools Appl 2018 Nov 28;78(11):15169-15211. [doi: 10.1007/s11042-018-6894-4]

32. Zhao W, Zou W, Chen JJ. Topic modeling for cluster analysis of large biological and medical datasets. BMC Bioinformatics 2014;15 Suppl 11:S11 [FREE Full text] [doi: 10.1186/1471-2105-15-S11-S11] [Medline: 25350106]

\section{Abbreviations}

LDA: Latent Dirichlet allocation

NLP: natural language processing

SNS: social network service

TF-IDF: term frequency-inverse document frequency 
Edited by G Eysenbach; submitted 08.04.20; peer-reviewed by R Poluru, M Lamba; comments to author 30.04.20; revised version received 15.06.20; accepted 07.07.20; published 13.08.20

Please cite as:

Min KB, Song SH, Min JY

Topic Modeling of Social Networking Service Data on Occupational Accidents in Korea: Latent Dirichlet Allocation Analysis J Med Internet Res 2020;22(8):e19222

URL: http://www.jmir.org/2020/8/e19222/

doi: $10.2196 / 19222$

PMID: $\underline{32663156}$

CKyoung-Bok Min, Sung-Hee Song, Jin-Young Min. Originally published in the Journal of Medical Internet Research (http://www.jmir.org), 13.08.2020. This is an open-access article distributed under the terms of the Creative Commons Attribution License (https://creativecommons.org/licenses/by/4.0/), which permits unrestricted use, distribution, and reproduction in any medium, provided the original work, first published in the Journal of Medical Internet Research, is properly cited. The complete bibliographic information, a link to the original publication on http://www.jmir.org/, as well as this copyright and license information must be included. 